\title{
INFRARED THERMOGRAPHY INSPECTION METHODS APPLIED TO THE TARGET ELEMENTS OF W7-X DIVERTOR
}

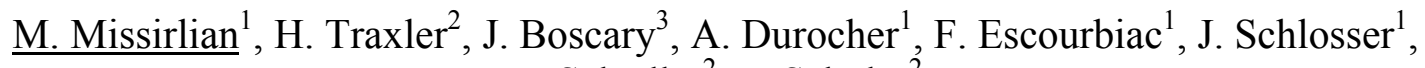 \\ B. Schedler ${ }^{2}$, P. Schuler ${ }^{2}$ \\ (1) Association Euratom-CEA, CEA/DSM/DRFC, CEA/Cadarache, F-13108 Saint Paul Lez Durance, France \\ (2) PLANSEE SE, Technology Center, A-6600 Reutte \\ (3) Max-Planck-Institut für Plasmaphysik, Euratom Association, Boltzmannstr. 2, D-85748 Garching, Germany
}

\begin{abstract}
The non-destructive examination (NDE) method is one of the key issues in developing highly-loaded plasma-facing components (PFCs) for a next generation fusion devices such as W7-X and ITER. The most critical step is certainly the fabrication and the examination of the bond between the armour and the heat sink. Two inspection systems based on the infrared thermography methods, namely, the transient thermography (SATIR-CEA) and the pulsed thermography (ARGUS-PLANSEE), are being developed and have been applied to the pre-series of target elements of the W7-X divertor. Results obtained from qualification experiences performed on target elements with artificial calibrated defects allowed to demonstrate the capability of the two techniques and raised the efficiency of inspection to a level which is appropriate for industrial application.
\end{abstract}

Keywords : W7-X, Plasma-Facing Component, Target Element, Non-Destructive Examination

\section{Introduction}

As heat exhaust capability and lifetime of highly-loaded plasma-facing components (PFCs) during in-situ operation are linked to the manufacturing quality, a set of non-destructive testing must be operated during R\&D and manufacturing phases. Within this framework, advanced non-destructive examination (NDE) methods are one of the key issues to achieve a high level of quality and reliability in the production of high heat flux (HHF) components for a next generation of fusion devices.

The W7-X target element (Fig. 1) is composed of 3D carbon fibre reinforced carbon composite tiles (CFC grade Sepcarb ${ }^{\circledR}$ NB31) assembled by Plansee SE by AMC ${ }^{\circledR}$ (Active Metal Casting) process to OFHC copper interlayer, and joined afterwards to a heat sink made of $\mathrm{CuCrZr}$ copper alloy. The cooling geometry is formed by four straight channels equipped with twisted tapes as turbulence promoters and connected by U-bends [1]. The type of target element (14 types in all) is characterized by its length (ranging from 250 to $595 \mathrm{~mm}$ ), and by its width (ranging from 50 to $61.5 \mathrm{~mm}$ ). For some element types, an additional width of $4.5 \mathrm{~mm}$ (so-called large side) has been introduced in the design (dashed line on the right side of the transversal view, see bottom part of Fig. 1) to allow the installation of diagnostics .

The reliable non-destructive evaluation of this multilayer component cannot be obtained by NDE methods -like ultrasonic and X-ray inspection, due to the porosity of the CFC material and to the very different acoustic impedances between the materials. Therefore, two infrared thermography inspection systems are being developed, namely, the transient thermography SATIR by CEA and the pulsed thermography of ARGUS by PLANSEE SE. They have been applied to judge the bonding quality of the target elements of W7-X divertor during the preseries activities.

The first step was the calibration of the system using full-scale pre-series target elements, in which calibrated defects of different sizes and geometries were manufactured at the interfaces between the tiles and the heat sink. It allowed the correlation between NDE results and defect topology. 
Finally, a cross-checking analysis, between HHF tests and these NDE methods by infrared thermography, correlated with finite elements analysis, allowed to propose a workable acceptance criterion for each method to be applied possibly during the series production of W7-X target elements. The application of these acceptance criteria during the pre-series fabrication is discussed in this paper.

\section{NDE infrared thermography methods}

The basic principle is that flaws in the bonds between materials modify the heat transfer in the component. To generate a heat transfer through the part to be inspected a temperature gradient needs to be established inside the component. The technique for the thermal activation defines the different NDE procedures.

\subsection{Transient thermography testing (SATIR)}

\section{Principle}

With Tore Supra Toroidal Pumped Limiter, CEA acquired a large experience in the inspection and acceptance processes of actively-cooled HHF elements armoured with CFC tiles using the infrared thermography SATIR (infrared test bed at CEA). The testing protocol is based on a thermal transient induced by an alternative hot $\left(95^{\circ} \mathrm{C}\right)$ and cold $\left(5^{\circ} \mathrm{C}\right)$ water flow in the heat sink structure. The design of the system allows to control several elements in parallel where the surface temperature of each tile of the elements is monitored by an infrared camera. The tile surface temperature transients are compared with those of an assumed defect free element (so-called reference) and the maximum of the temperature difference (so-called DTref_max) is stored for each tile [2]. A bonding flaw between heat sink and armour will be detected by a high value of DTref_max. The infrared thermography SATIR was used for the acceptance of the $\sim 700$ finger elements of the Toroidal Pumped Limiter. It gives a quantitative information on the global thermal efficiency of the component prior to its installation into the vacuum vessel of a fusion machine. This facility permits also through an acceptance test based on a reference element to detect variability within the produced elements through the reception of the whole production.

\section{Results of NDE performed on mock-ups with artificial defects}

Two mock-ups (no. 4B-1V and no. 4B-7V) with artificial calibrated defects were manufactured during the pre-series phases and controlled on SATIR before high heat flux testing (pre-HHF) [3]. The geometry and location of these artificial defects was based on the experience gained during the fabrication of the target elements: defects always located at the lateral edge of the elements, corner or strip geometries. The defects were manufactured in $\mathrm{CFC}$ at the interface between $\mathrm{CFC}$ and the copper interlayer, except for tile 1 at the interface between copper and $\mathrm{CuCrZr}$.

Fig. 2 shows an IR image of the DTref_max value cartography during the cooling phase for the $4 \mathrm{~B}-1 \mathrm{~V}$ target element. The main outcomes point out that strip defects up to $4 \mathrm{~mm}$ and corner defects up to $6 \times 6 \mathrm{~mm}$ are reliably detectable with SATIR. The correlation between the experimental measurements and the defect size is defined by the graphs which gives the measured DTref_max as a function of the size of the calibrated defects. Thereafter, the measurements performed on theoretically free-defect tiles allowed to evaluate the limit of SATIR sensibility, namely $1.5^{\circ} \mathrm{C}$ for the small side (to be applied to each side of the standard elements) and $2^{\circ} \mathrm{C}$ for the large side (to be applied to one side of the diagnostic elements). The thermal-hydraulic conditions used during the SATIR tests are: water velocity $8 \mathrm{~m} / \mathrm{s}$, pressure 10 bars.

\subsection{Pulsed thermography (ARGUS)}




\section{Principle}

In case of pulsed thermography, the temperature gradient necessary for NDE is established by a single powerful flash of light which is directed towards the surface of the CFC tiles. The light source of the ARGUS setup releases an energy of $12 \mathrm{~kJ}$ within $0,1 \mathrm{~s}$. The cool down of the CFC surface is recorded applying an infrared camera with good temperature resolution (NETD@20 $20^{\circ} \mathrm{C}=20 \mathrm{mK}$ ) and high image frequency (up to $150 \mathrm{~Hz}$ ). For each pixel, a frequency analysis based on the fast Fourier transform algorithm (FFT) is calculated on the cool down behaviour after the flash. The defect detection is not based on the power spectrum of the FFT, but on the phase-shift of the basic frequency of the FFT (f1) and its higher harmonics $(\mathrm{f} 2, \mathrm{f} 3, \mathrm{f} 4, \ldots)$. The basic frequency ( $\mathrm{fl}$ ) is adjusted to obtain a penetration depth reaching the $\mathrm{Cu} / \mathrm{CuCrZr}$ interface whereas the penetration of the first harmonic frequency (f2) is limited to the $\mathrm{CFC} / \mathrm{Cu}$ interface. This means that the phase image of each frequency addressed by the FFT carries information about the depth of the defect detected.

The method can be applied on the component without using the cooling system and is implemented at a rather advanced stage of production. Therefore the bonding quality can be assessed at any stage of the manufacturing route, allowing an earlier detection to assess the quality of the bond. This is an important feature for PLANSEE SE as a manufacturer of divertor components to develop a reliable quality-control tool, which can be efficiently integrated in an industrial process. Since there is no need for a reference element, the test setup is quite simple and the evaluation of the data for rating the bonding quality of the component under inspection is finished within a few seconds.

\section{Results of NDE performed on mock-ups with artificial defects}

The defect detection capabilities of the inspection method are demonstrated for the test body component no. 4B-1V which has been tested by ARGUS. In Fig. 3 the phase images of the basic frequency (f1) and the first harmonic frequency (f2) are shown. Defects lead to lower phase shift values in both images. For defect detection the areas showing a minimum phase shift are analyzed. The penetration of the signal depends on the FFT frequency component selected. The higher the frequency, the smaller the penetration depth of the derived phase image [4]. Therefore the artificial defect in the $\mathrm{Cu} / \mathrm{CuCrZr}$ interface (see tile no. 1) gives a stronger signal in the phase image of frequency $\mathrm{fl}$ which has the deeper penetration. The areas without artificial defects in component 4B-1V appear quite homogeneous apart from some non-artificial defects in the component. For an estimation of the repeatability of the measurements, 10 measurements on component $4 \mathrm{~B}-1 \mathrm{~V}$ performed on different days were analyzed. The scatter of the minimum phase shift in phase image of frequency f1 measured on tile no. 1 is $0,002 \mathrm{rad}$, which represents less than $0.5 \%$ of the acceptance phase shift threshold (see below from Table 2).

\section{Comparison of NDE results with HHF tests}

The results of NDE performed on target elements with artificial calibrated defects, were compared with the high heat flux tests to correlate the joint defect size detected with NDE to the thermal response obtained under heat loading. HHF testing was carried out in JUDITH electron beam facility [5] with different experimental conditions to achieve the nominal heat flux expected during operation in W7-X. As it is pointed out in Fig. 4, calibrated defects up to $6 \mathrm{~mm}$ were systematically detected on tiles with AMC defects.

\subsection{Comparison with SATIR/Definition of an acceptance criterion}

The experimental results have been compared to 3D finite element calculations. The purpose was to correlate the HHF test results with the NDE infrared thermography examination as a function of the $\mathrm{CFC} / \mathrm{Cu}$ topology defects. 
It is assumed that the definition of an acceptance limit on the SATIR test bed is equivalent to accept a limited overheating under normal operation $\left(10 \mathrm{MW} / \mathrm{m}^{2}\right.$ in steady state). A maximum local overheating (i.e. a local increase of surface temperature with respect to the average temperature) limited to $30 \%$ for the small side and $40 \%$ for the large side with respect to the average temperature was evaluated from experimental observations in JUDITH. Finally, the maximal acceptable defect, correlated to its response under infrared examination, allows to define a cut-off value in terms of DTref_max on SATIR test bed (Fig. 5). This cut-off value determined for each type of target elements (namely, the standard and the diagnostic elements) is reported in the Table 1. Taking into account the uncertainties due to the background noise of the SATIR device and the industrial factors like the materials properties and/or geometrical tolerances, this value allows to define a workable acceptance criteria to be applied on flat tiles with two threshold values (Fig. 6):

- The first one is the threshold value below which the examined tile is accepted without any question (color-code: white);

- The second one is the threshold value above which the examined tile is rejected (colorcode: black);

- Between the two threshold values (color-code: grey), the examined tile is questionable .

\subsection{Comparison with ARGUS/Definition of an acceptance criterion}

For each phase image a threshold value of phase shift has been defined in order to rate the bonding quality of each tile. The phase shift values of the criterion were defined in order to obtain as good correlation to the HHF test as possible. The definition of the acceptance criterion is given in Table 2. The application of the acceptance criterion to the phase images gives the rated phase images which are shown in Fig. 7 for component 4B-1V containing artificial defects (see tile no. 1, 2, 3, 4, 7, 8, 9 and 10) and manufacturing defects (see tile no. 5 and 6). The shape of all artificial defects is displayed quite well in at least one of the two phase images indicating that they can be discriminated by the acceptance criterion described in Table 2. Of course the defect in the $\mathrm{Cu} / \mathrm{CuCrZr}$ interface (see tile no. 1 and 2) appears smaller than the corresponding defect of the same size in the $\mathrm{CFC} / \mathrm{Cu}$ interface (see tile no. 2 and 3). The detection of the $2 \mathrm{~mm}$ strip defect in the $\mathrm{CFC} / \mathrm{Cu}$ interface (see tile no. 7 ) is best in phase image of frequency $f 2$ with the smaller penetration depth. The final rating of each tile is based on both phase images. Attempts to rate an average image of the two phase images did not lead to a comparable correlation of the pre-HHF ARGUS inspection to the HHF result. If in at least one of the phase images a black spot occurs the tile is rejected. If only grey areas appear in at least one phase image the tile is rated as questionable.

\section{Application to target elements}

To check the reliability of both NDE infrared thermography methods, the acceptance criteria defined for each method were applied to W7-X pre-series target elements. The results were benchmarked with HHF tests to assess the detection ability of defects at the interface between tiles and heat sink.

8 pre-series target elements of type $4 \mathrm{~S}$ with $10 \mathrm{CFC}$ tiles per elements, that is to say a maximum total amount of 80 tiles, were inspected on SATIR and ARGUS test beds, screened at $\sim 6 \mathrm{MW} / \mathrm{m}^{2}$ on GLADIS facility [6], then fatigued at $\sim 10 \mathrm{MW} / \mathrm{m}^{2}(10$ seconds pulseduration per cycle) up to 100 cycles.

Correlation between the thermographic examinations and the initial screening GLADIS tests is very acceptable (Fig. 8). On the other hand, the correlation between failure (i.e. progressing hot spot) under thermal-cycling and initial screening is low. Large defects at the bond, which appeared during the first HHF cycles, were predicted by the two NDE methods (i.e. DTref_max $>4.5^{\circ} \mathrm{C}$ for SATIR and $\operatorname{Min} \Phi<0,734$ rad-phase image $1 / \operatorname{Min} \Phi<0,554$ rad- 
phase image 2 for ARGUS). For tiles fulfilling the acceptance criteria associated at each NDE method, about $26 \%$ showed some degradation during the fatigue tests. Among these, a few percent of tiles were detected suspicious during GLADIS screening at $\sim 6 \mathrm{MW} / \mathrm{m}^{2}$.

Considering the present available database, the criteria of rejection associated to each non destructive method are reliable and validated (100\% of the rejected tiles after SATIR and ARGUS tests, were also detected as defective on GLADIS). On the other hand, the criteria of derogation requires still some improvements. The low level of agreement observed between the NDE methods or the screening GLADIS tests, and the HHF cycling may probably caused by the propagation of defects during the HHF test. Systematic comparisons of the thermography inspection before and after the HHF test has started, underlining this behaviour on several tiles. However, some studies are still needed to better understand the detailed failure processes and to obtain information about defect typology. In addition, the SATIR and ARGUS tests have also to be improved and optimized as soon as a broader data base will be available. Furthermore, some improvements in the design may also enable an increase of the agreement between NDE methods and HHF tests.

\section{Conclusion}

In the plasma facing components fields, there is a need of advanced NDE methods to demonstrate that defects, which can impair the component performances and/or integrity during operation, can be identified, located and sized in a reliable way. In this frame, two promising infrared thermography methods, the transient (SATIR) and the pulsed (ARGUS) thermography, respectively, have been used during the pre-series activities of W7-X divertor target elements.

Transient thermography is a reliable NDE method, which gives a quantitative information on the global thermal efficiency of the component prior to its installation into a fusion machine. This facility permits also through an acceptance test based on a reference element to detect variability within the produced elements during the whole production.

With the application of pulsed thermography, a method is available which enables defect detection at any stage of the production. This information leads to the identification of processes which are critical for the quality of the components.

Results obtained from qualification experiences performed on mock-ups with artificial calibrated defects, demonstrated the capability of the two methods to detect different types of defect in the size range 2-4 $\mathrm{mm}$. These tests enabled to define a workable acceptance criterion associated to each method valuable for the serial production of the W7-X divertor target elements. The application on pre-series target elements, showed a good correlation with the initial screening at $6 \mathrm{MW} / \mathrm{m}^{2}$. However, some defects -non-detected as defective during NDE- appeared or propagated during cycling at nominal heat fluxes $\left(10 \mathrm{MW} / \mathrm{m}^{2}\right.$ in steady state). This behaviour led to an extension of the pre-series activities to improve the safety margin in the design and to achieve a successful series production of the $\mathrm{W} 7-\mathrm{X}$ divertor target elements. 


\section{References}

[1] J. Boscary et al., Applied technologies and inspection for the W7-X pre-series target elements, Journal of Fusion Engineering and Design 75-79 (2005), pp. 451-455

[2] A. Durocher et al., Interface quality control by infrared thermography measurement, Proc. $15^{\text {th }}$ World Conference on NDT, Roma, Italy, October 15-21 (2000)

[3] M. Missirlian et al., Commissioning tests of W7-X target elements-Analysis in view of the acceptance criteria definition, CEA report, DSM/DRFC/SIPP/2005-002, March 2005

[4] G. Busse, Pulsed phase thermography - Theory, Nondestructive Testing Handbook, Volume 3, Infrared and Thermal Testing, 3rd Edition, American Society for Nondestructive Testing (2001), pp. 313-316

[5] M. Rödig et al., Testing of actively cooled mock-ups in several high heat flux facilitiesAn international round robin test, , Journal of Fusion Engineering and Design 75-79 (2005), pp. 303-306

[6] H. Greuner et al., Design, performance and construction of a $2 \mathrm{MW}$ ion beam test facility for plasma facing components, Journal of Fusion Engineering and Design 75-79 (2005), pp. 345-350 


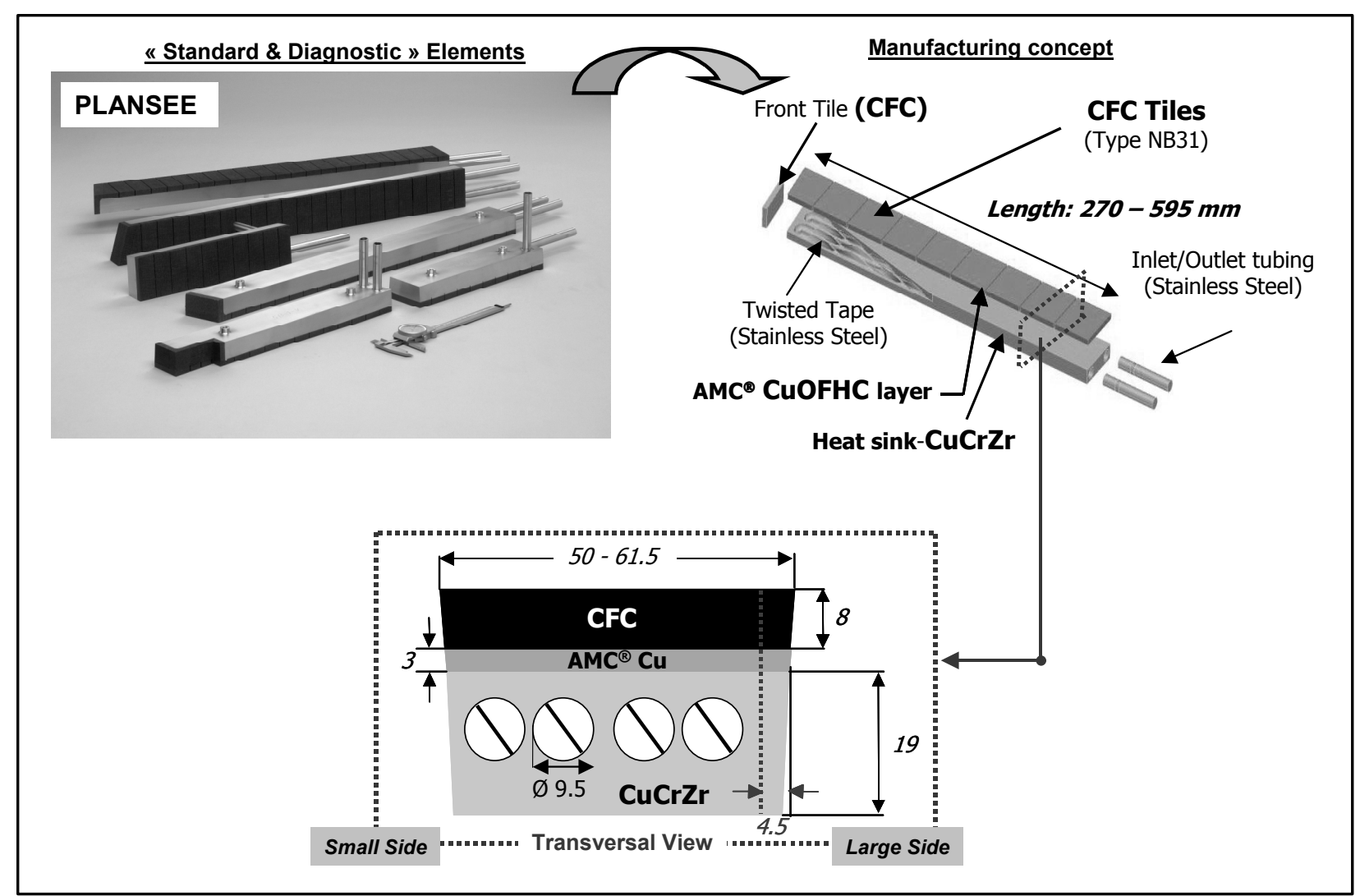

Figure 1. Target Elements of W7-X Divertor 


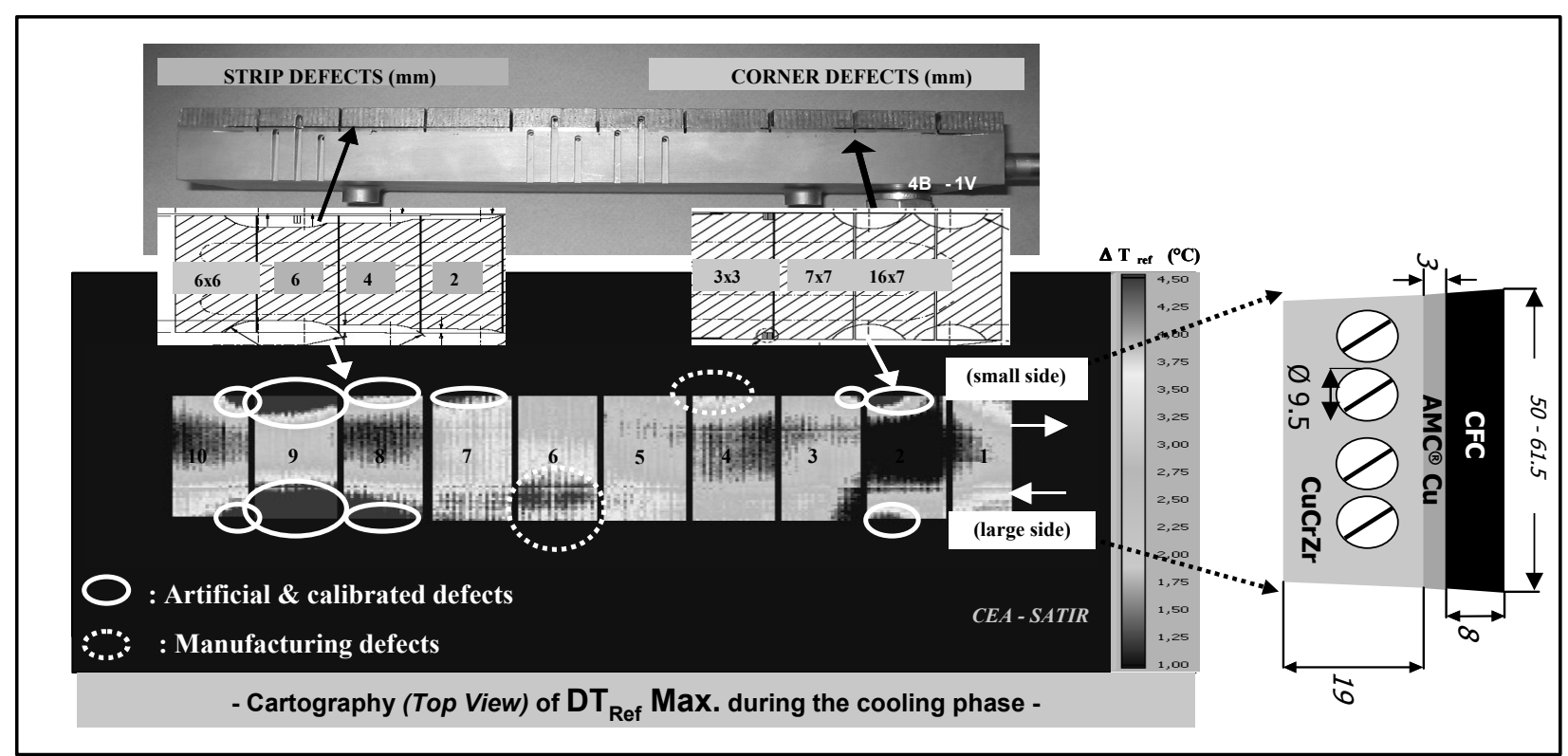

Figure 2. SATIR results: 4B-1V element (with artificial defects)

- IR cartography of DTref_max during the cooling phase - 

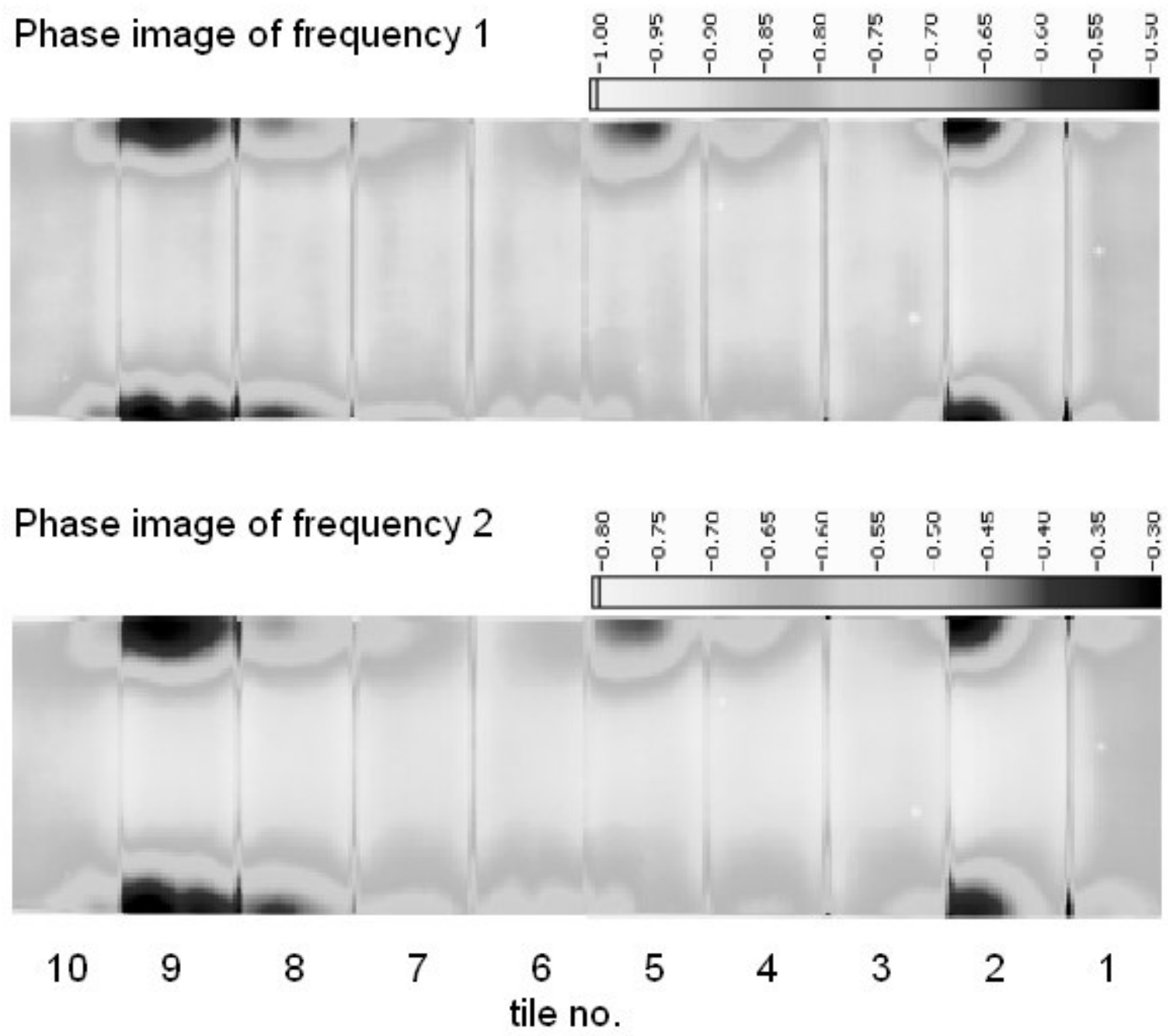

Figure 3. ARGUS results: 4B-1V element (with artificial defects)

- Phase image of frequency 1 (basic frequency f1) and frequency 2 (first harmonic f2) - 


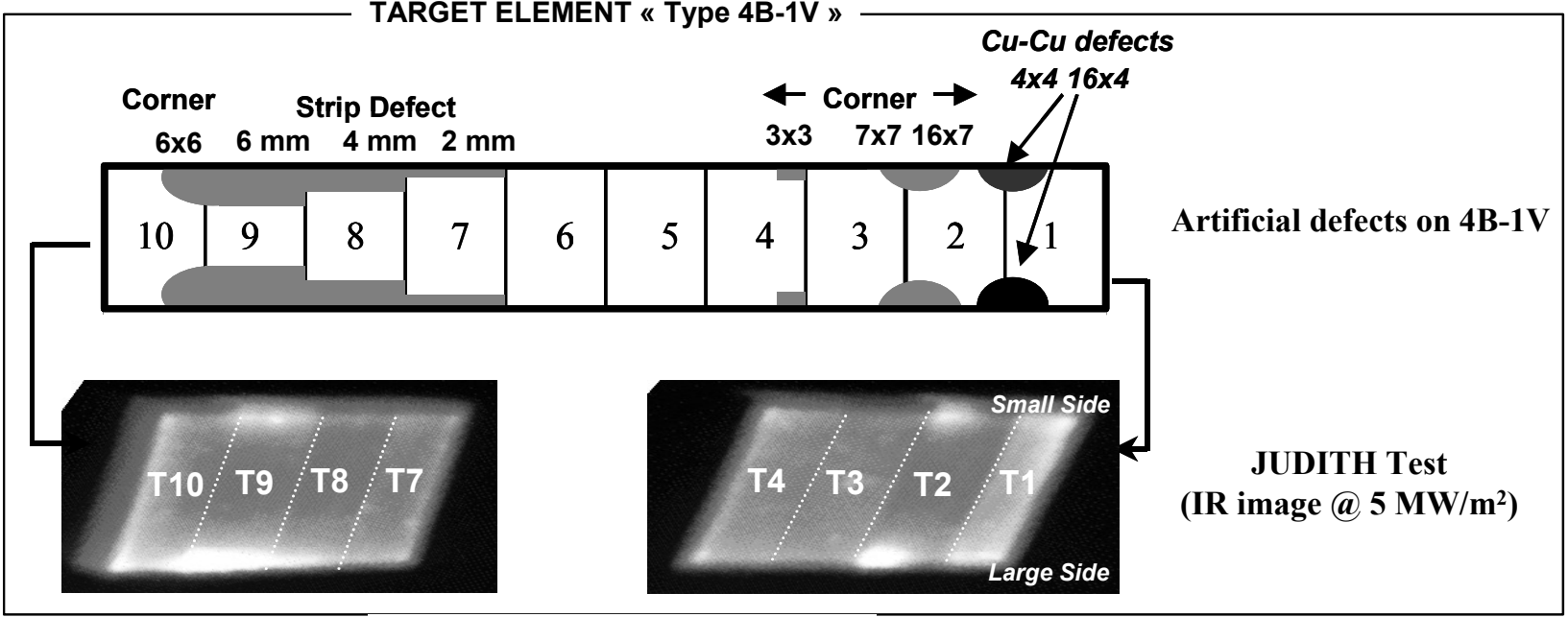

TARGET ELEMENT « Type 4B-7V »

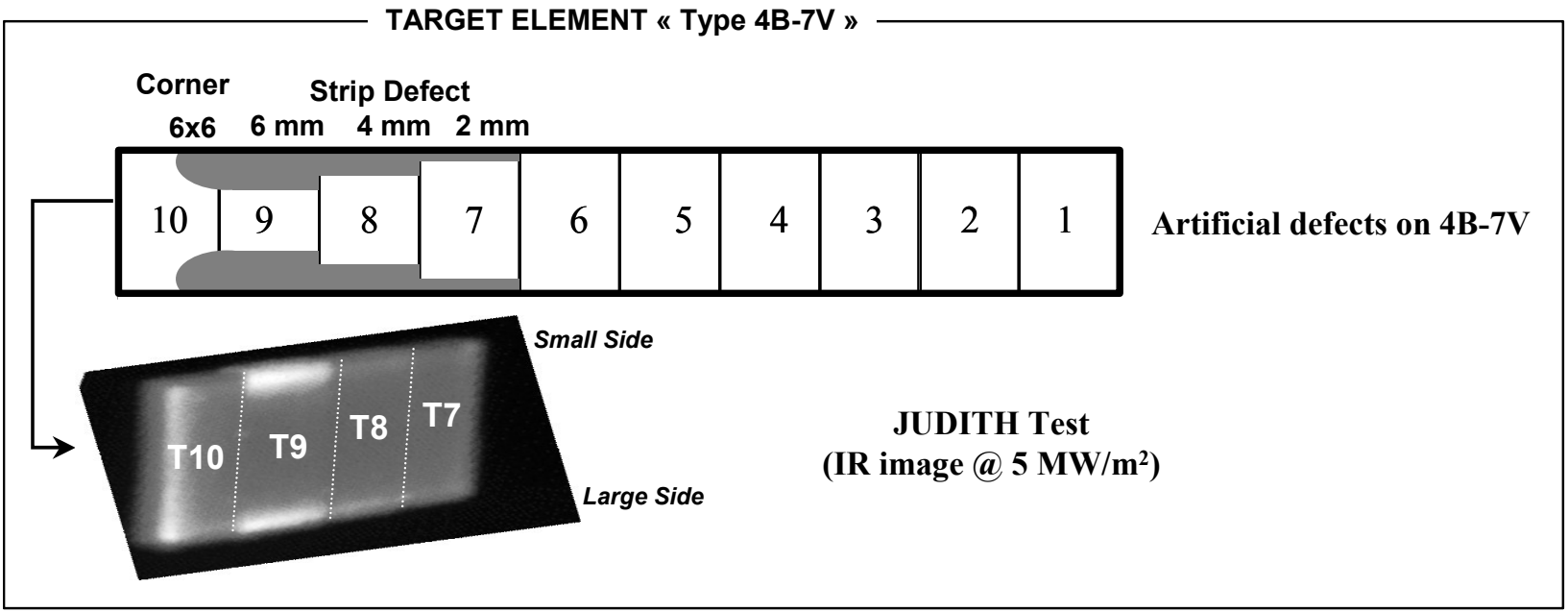

Figure 4. HHF Testing: Partial infrared view of tiles with artificial \& calibrated defects during JUDITH screening @ $5 \mathrm{MW} / \mathrm{m}^{2}$ 


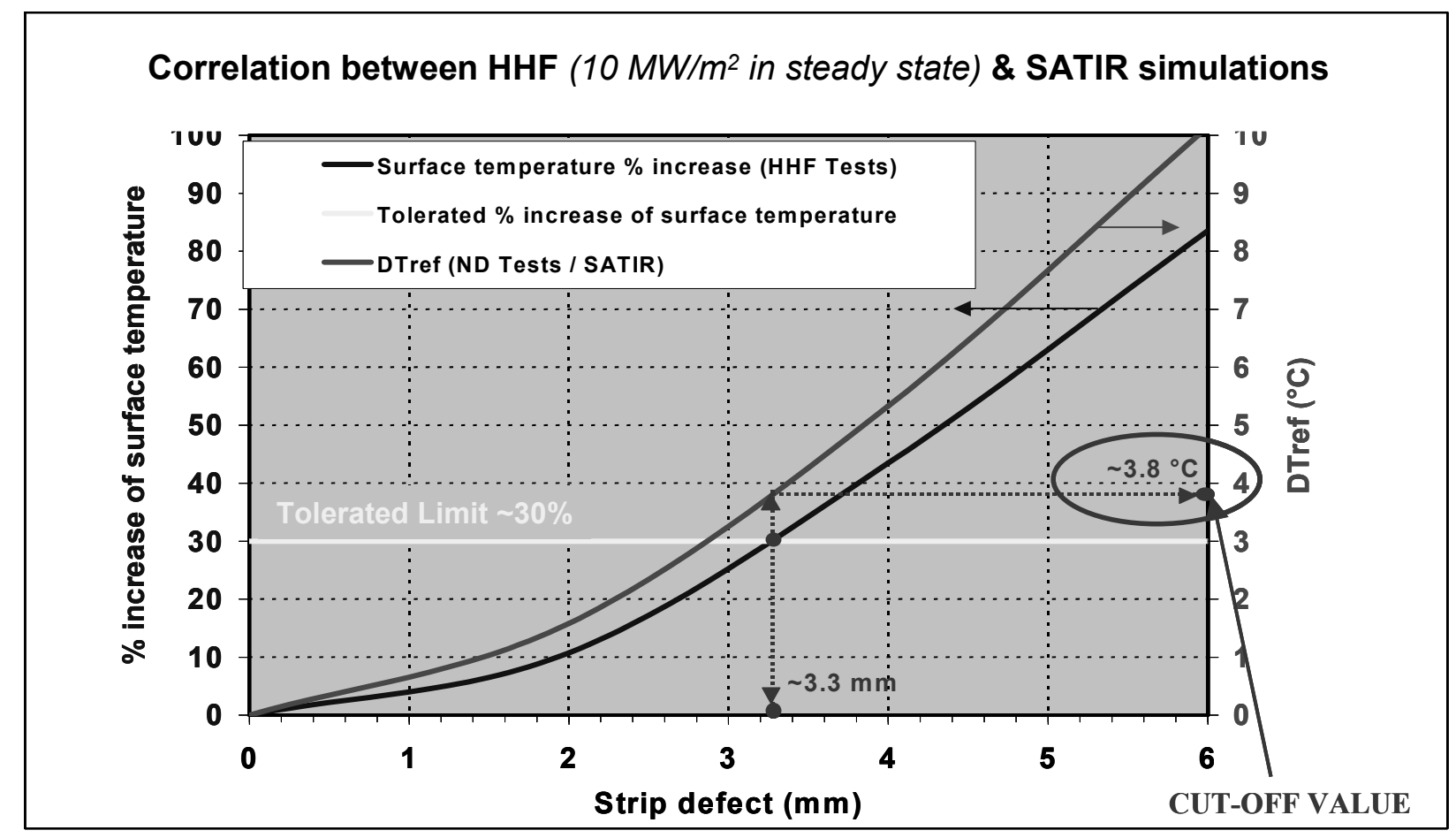

Figure 5. Correlation between high heat flux testing and SATIR examinations - "Cut-Off value" determination for the small side (i.e. standard elements) 

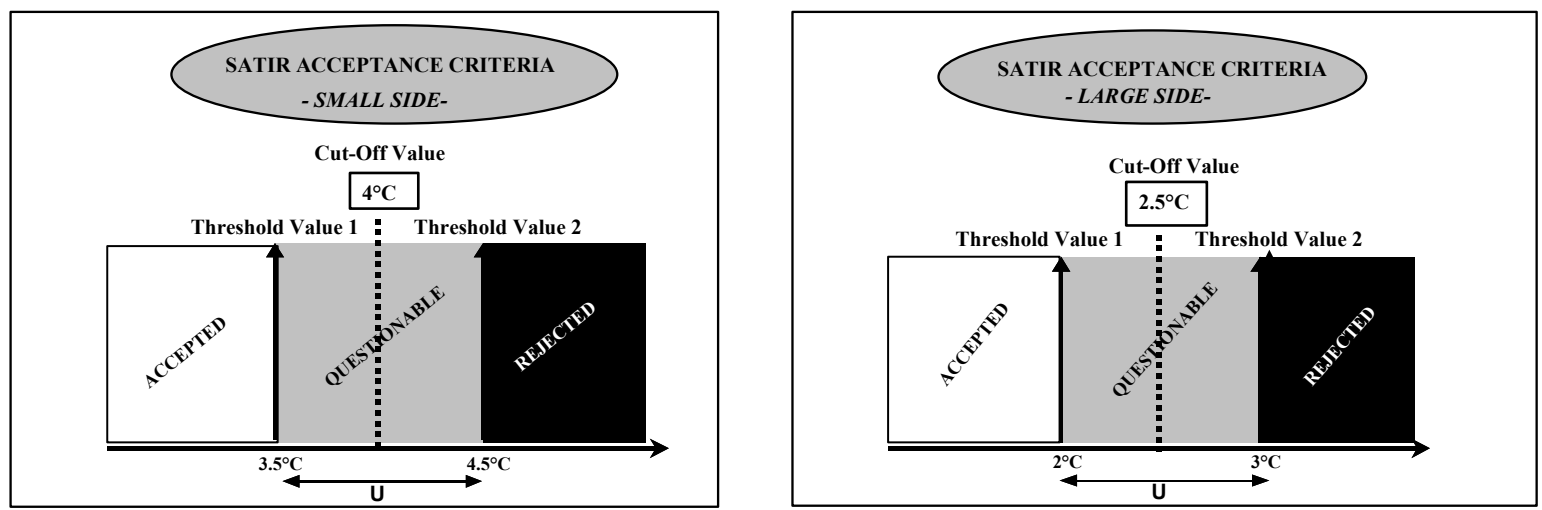

U : Uncertainties due to background noise of the (SATIR) device \& the engineering factors

Figure 6. SATIR Acceptance Criteria for the small side (i.e. standard element) and large side (i.e. diagnostic element) of target elements 
Rated phase image of frequency 1
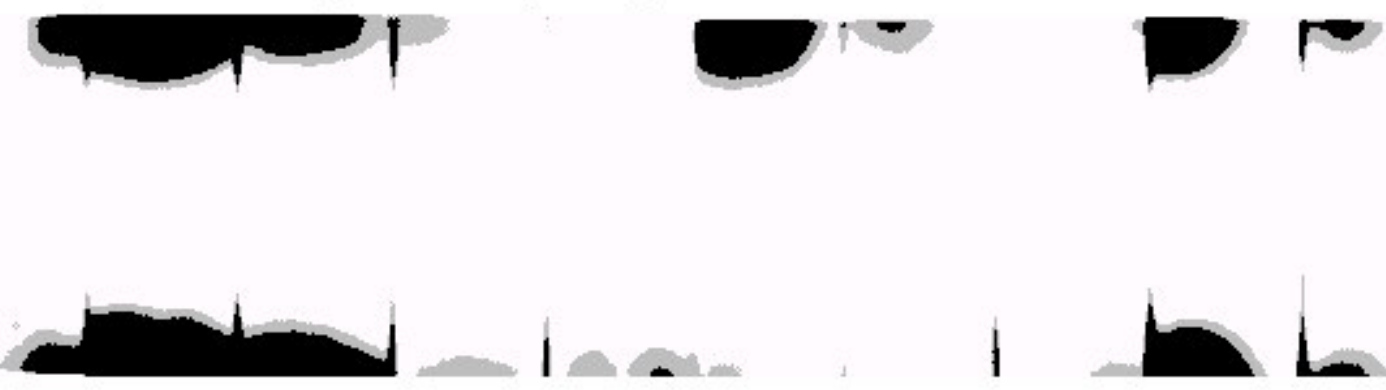

1

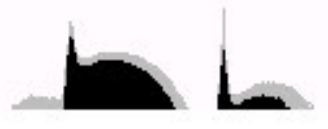

Rated phase image of frequency 2
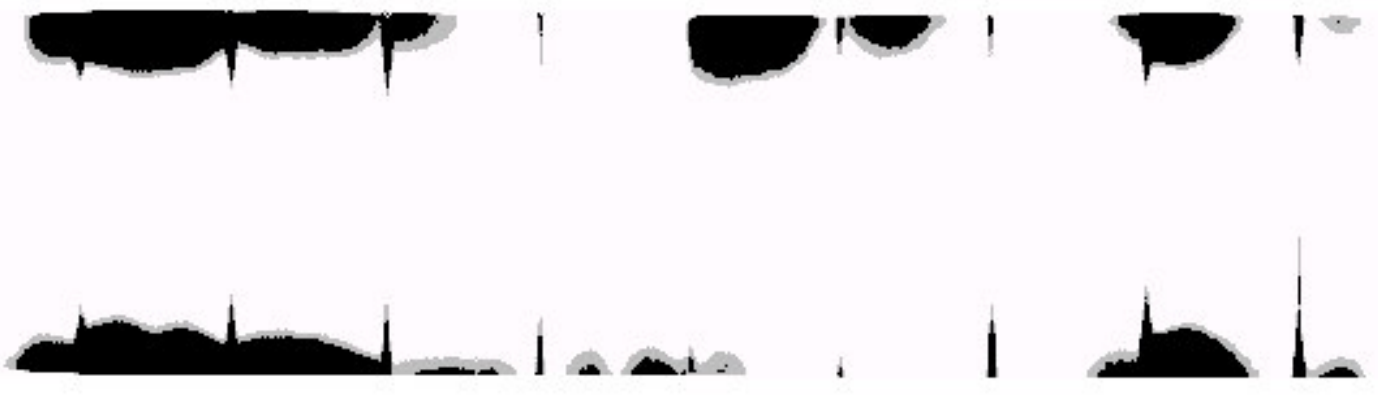

109

8

7

$6 \quad 5$

4

32

1 tile no.

not accepted

Figure 7. ARGUS results: 4B-1V element (with artificial defects)

- Rated phase images of frequency 1 (basic frequency f1) and frequency 2 (first harmonic f2) 


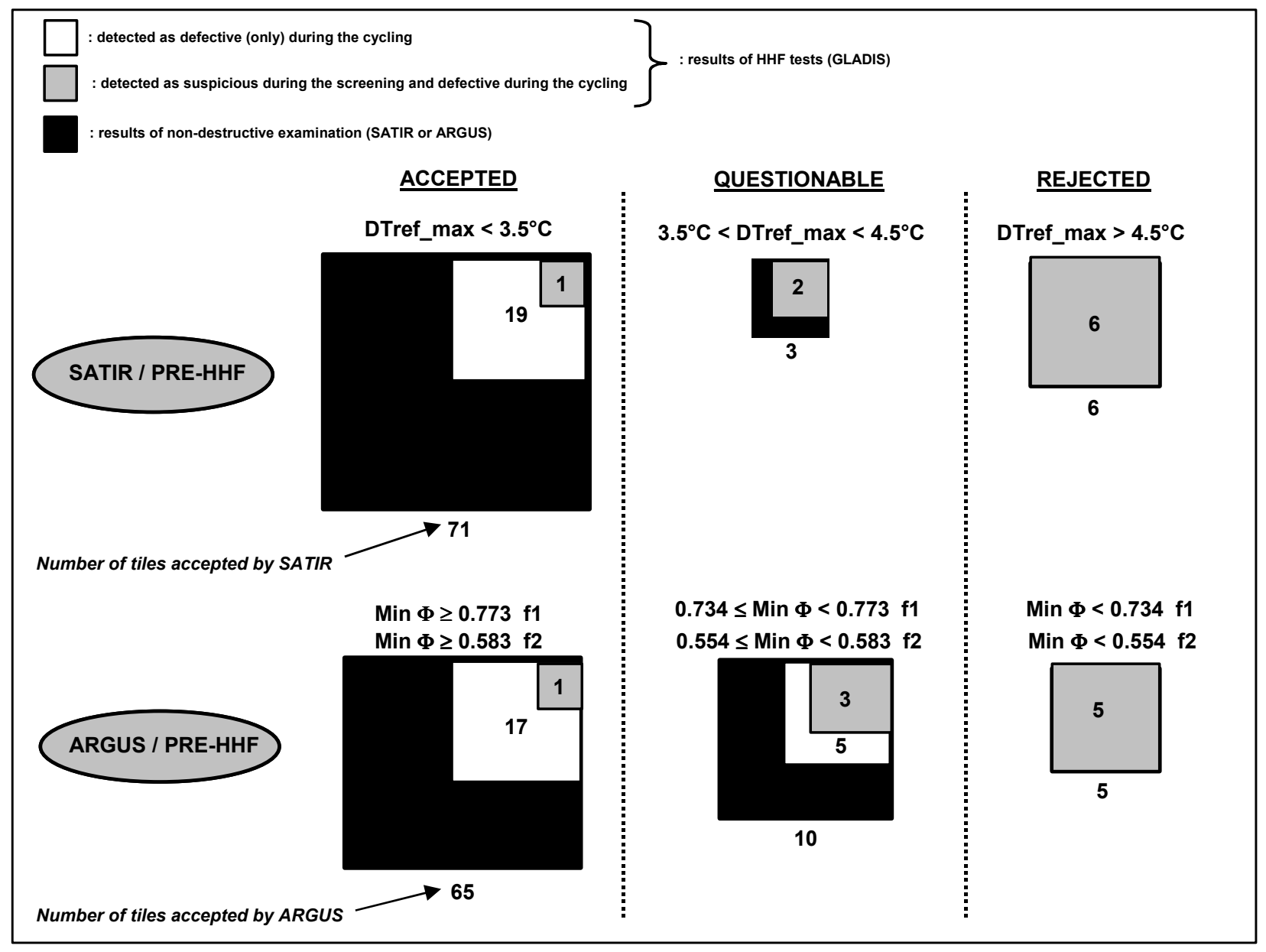

Figure 8. Statistic repartition on a population of 80 tiles (Batch \#3) from the industrial manufacture characterized on SATIR and ARGUS, then screened and fatigued at GLADIS 
Table 1. Cut-Off value in terms of DTref_max (SATIR) for each type of defect

\begin{tabular}{|c|c|c|c|c|}
\hline & \multicolumn{2}{|c|}{ STRIP DEFECT } & \multicolumn{2}{|c|}{ CORNER DEFECT } \\
\hline & Small Side & Large Side & Small Side & Large Side \\
\hline $\begin{array}{c}\text { DTref } \\
\text { (Cut-Off value) }\end{array}$ & $\begin{array}{c}\sim 3 . \mathbf{8}^{\circ} \mathbf{C} \\
\text { (see Fig. 5) }\end{array}$ & $\sim 2.3^{\circ} \mathrm{C}$ & $\sim 4.2^{\circ} \mathrm{C}$ & $\sim 2.7^{\circ} \mathrm{C}$ \\
\hline Size of defect & $3.3 \mathrm{~mm}$ & $3.4 \mathrm{~mm}$ & $5.5 \times 5.5 \mathrm{~mm}$ & $6 \times 6 \mathrm{~mm}$ \\
\hline SATIR Sensibility & $1.5^{\circ} \mathrm{C}$ & $2^{\circ} \mathrm{C}$ & $1.5^{\circ} \mathrm{C}$ & $2^{\circ} \mathrm{C}$ \\
\hline
\end{tabular}


Table 2. Acceptance criterion for the ARGUS test (the phase shift values are given in rad.)

\begin{tabular}{|c|c|c|c|}
\cline { 2 - 4 } \multicolumn{1}{c|}{} & accepted & questionable & rejected \\
\hline phase image 1 & $\Phi ? \mathbf{0 , 7 7 3}$ & $\mathbf{0 , 7 3 4} \boldsymbol{?} \Phi<\mathbf{0 , 7 7 3}$ & $\Phi<0,734$ \\
\hline phase image 2 & $\Phi ? \mathbf{0 , 5 8 3}$ & $\mathbf{0 , 5 5 4} \boldsymbol{?} \Phi<\mathbf{0 , 5 8 3}$ & $\Phi<\mathbf{0 , 5 5 4}$ \\
\hline
\end{tabular}

